\title{
KLOE Results on Hadron Physics and Perspectives for KLOE-2
}

\author{
Simona Giovannella ${ }^{1, \star}$ on behalf of the KLOE-2 Collaboration \\ ${ }^{1}$ Laboratori Nazionali di Frascati dell'INFN, Frascati, Italy
}

\begin{abstract}
Recent results obtained with KLOE data on light meson decays, together with perspectives for the current KLOE-2 run, are reviewed.
\end{abstract}

\section{Introduction}

The KLOE-2 experiment aims to enlarge and extend the KLOE physics programs with a larger data set and an upgraded detector. The KLOE detector [1] has collected $2.5 \mathrm{fb}^{-1}$ at the $e^{+} e^{-}$collider DAФNE [2], running at the peak of the $\phi$ resonance, $M_{\phi} \sim 1020 \mathrm{MeV}$. An off-peak run provided also 250 $\mathrm{pb}^{-1}$ at $1 \mathrm{GeV}$. The detector consists of a large cylindrical drift chamber [3] and an electromagnetic calorimeter [4], surrounded by a magnetic field of $0.52 \mathrm{~T}$. The trigger [5] uses information from both the calorimeter and the drift chamber. Collected data are analyzed by an event classification filter [6], which selects and streams various categories of events in different output files.

A new beam crossing scheme, allowing for a reduced beam size and increased luminosity, is now operating at DAФNE [7]. The KLOE-2 detector is collecting $e^{+} e^{-}$collision data at center of mass energy equal to $M_{\phi}$, with the aim of a final data set of $\sim 5 \mathrm{fb}^{-1}$. Four tag stations [8], the High Energy Taggers (HET) and the Low Energy Taggers (LET), have been installed to detect electrons and positrons from the reaction $e^{+} e^{-} \rightarrow e^{+} e^{-} \gamma^{*} \gamma^{*} \rightarrow e^{+} e^{-} X$, to investigate $\gamma^{*} \gamma^{*} \rightarrow \pi^{0} / \pi \pi / \eta / \eta \pi$ physics at the $\phi$ resonance. An inner tracker [9] has been installed between the beam pipe and the inner wall of the DCH to increase the acceptance for low transverse momentum tracks and improve charged vertex reconstruction. Photon detection has been improved by means of a small crystal calorimeter in the forward direction [10] and of a tungsten-scintillating tile sampling device instrumenting the low-beta quadrupoles located inside the detector [11]. A detailed description of the extended experimental physics program can be found in Ref. [12].

In this paper recent results obtained with KLOE data on the decay dynamics of $\eta \rightarrow \pi^{+} \pi^{-} \pi^{0}$ (Sec. 2) and on $\phi \rightarrow \eta e^{+} e^{-}, \phi \rightarrow \pi^{0} e^{+} e^{-}$transition form factors (Sec. 3) are reported. Perspectives on light meson spectroscopy at KLOE-2 are discussed in Sec. 4, while $\gamma \gamma$ physics at KLOE and KLOE-2 is described in Sec. 5.

\section{Decay dynamics of $\boldsymbol{\eta} \rightarrow \pi^{+} \pi^{-} \pi^{0}$}

The $\eta \rightarrow \pi^{+} \pi^{-} \pi^{0}$ decay is fully dominated by the isospin violating part of the strong interactions. A high precision measurement of the $\eta \rightarrow \pi^{+} \pi^{-} \pi^{0}$ decay dynamics allows to access the light quark mass

\footnotetext{
^e-mail: simona.giovannella@lnf.infn.it
} 

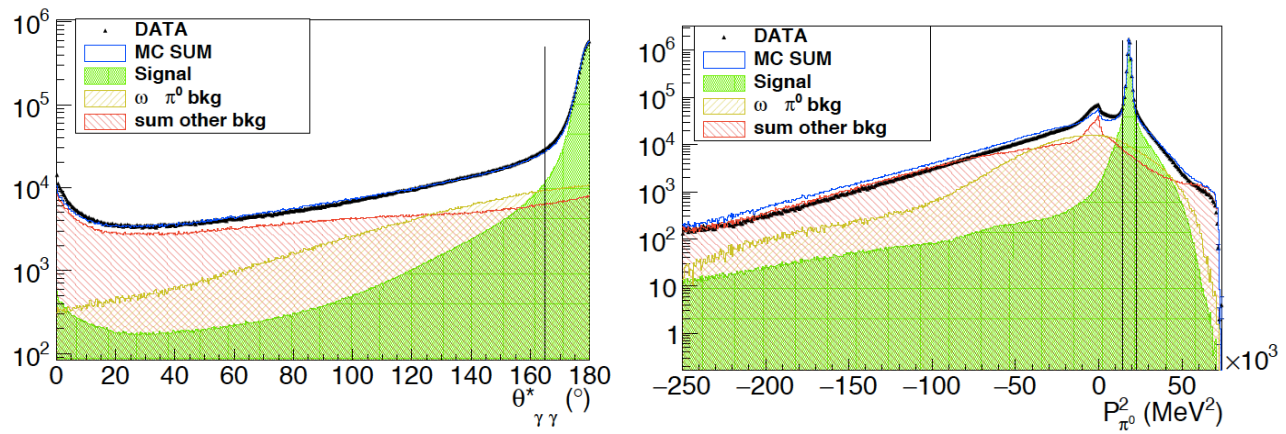

Figure 1. Dalitz plot analysis of $\eta \rightarrow \pi^{+} \pi^{-} \pi^{0}$ : data-MC comparison for the missing mass squared of the $\pi^{0}$ (left) and the opening angle between photons in the $\pi^{0}$ rest frame (right). The vertical lines represent the selection cuts.

difference [13]. The Dalitz plot density is commonly described by a polinomial expansion in the $X$ and $Y$ variables

$$
\begin{aligned}
X & =\sqrt{3}\left(T_{+}-T_{-}\right) / Q_{\eta}, \\
Y & =3 T_{0} / Q_{\eta} .
\end{aligned}
$$

In these relations, $T$ is the kinetic energy of the pions in the $\eta$ rest frame while $Q_{\eta}=m_{\eta}-2 m_{\pi^{ \pm}}-$ $m_{\pi^{0}}$. The squared amplitude of the decay can then be extracted with a fit:

$$
|A(X, Y)|^{2} \simeq N\left(1+a Y+b Y^{2}+c X+d X^{2}+e X Y+f Y^{3}+g X^{2} Y+h X Y^{2}+l X^{3}+\ldots\right)
$$

In 2008, the KLOE experiment measured for the first time the Dalitz Plot parameters up to the term $f$ (KLOE08) [14]. These results have been recently confirmed, although with less precision, by the WASA and BESIII experiments $[16,17]$. A new measurement has been carried out with KLOE data (KLOE16), with an independent and $\sim 4$ times larger data set $\left(1.7 \mathrm{fb}^{-1}\right)$, a new analysis scheme and an improved Monte Carlo simulation, providing with improved accuracy the parameters of the decay matrix [15].

In KLOE, light mesons are produced via radiative decays of the $\phi$ and are tagged by identifying the recoil monochromatic photon, $E_{\text {recoil }}$. The $\eta \rightarrow \pi^{+} \pi^{-} \pi^{0}$ selection requires two additional prompt neutral clusters from the $\pi^{0}$ and two tracks with opposite curvature in the drift chamber pointing to the IP. Decay kinematics is then exploited to constrain $E_{\text {recoil }}$ and to assign photons to $\pi^{0}$. Background scaling factors are obtained by fitting data with MC distribution for two variables: the missing mass squared of the $\pi^{0}$ and the opening angle between photons in the $\pi^{0}$ rest frame (Fig. 1). Cuts on these variables are used to reduce the background contamination. The resulting efficiency for signal events is $37.6 \%$, with a background contamination less than $1 \%$.

The resulting Dalitz plot density has been fitted, after background subtraction, with the complete third order polinomial expansion of Eq. (3), folded with smearing matrix and analysis efficency. Bin size is about three times the $X, Y$ resolution. Fitting with the whole polinomial expansion, the $c, e$, $h$ and $l$ parameters are consistent with zero, as expected from $C$-invariance. Fixing them to zero and comparing with the previous KLOE measurement (Tab. 1), the statistical uncertainty is reduced by about a factor of two, while improving also the systematic uncertainties, which are in some cases reduced by a factor of $2 \div 3$. The major improvement in the systematic uncertainties comes from the analysis of the effect of the event classification with an unbiased prescaled data sample. When 


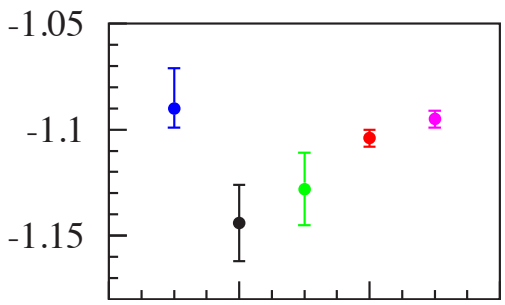

a

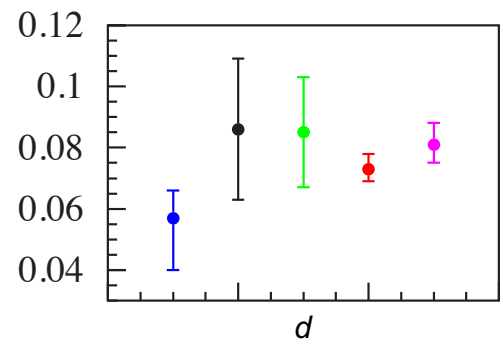

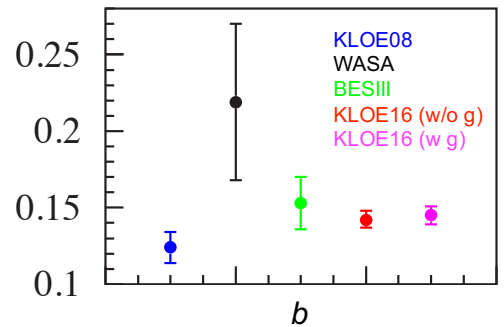

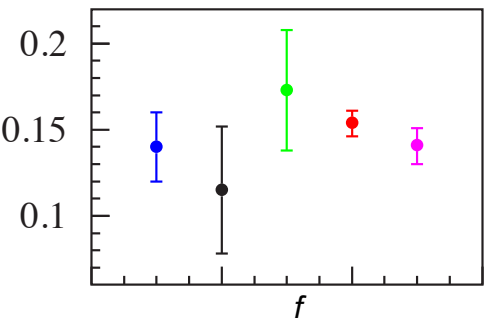

Figure 2. Comparison of $a, b, d, f$ parameters extracted from the fit to the Dalitz plot distribution of $\eta \rightarrow \pi^{+} \pi^{-} \pi^{0}$ decay. KLOE results $[14,15]$ are compared with recent measurements from WASA [16] and BESIII [17].

the $g$ parameter is included in the fit, its value is different from zero at $3 \sigma$ level, improving the $\chi^{2}$ probability from $24 \%$ to $56 \%$. Comparison of KLOE, WASA and BESIII results is reported in Fig. 2, showing a good agreement among experimental results, with KLOE16 being the most precise one.

Table 1. Fit results for $\eta \rightarrow \pi^{+} \pi^{-} \pi^{0}$ Dalitz plot analysis, compared to previous KLOE measurement.

\begin{tabular}{cccccc}
\hline & $a$ & $b$ & $d$ & $f$ & $g$ \\
\hline KLOE08 & $-1.090(5)\left({ }_{-19}^{+8}\right)$ & $0.124(6)(10)$ & $0.057(6)\left(_{-16}^{+7}\right)$ & $0.14(1)(2)$ & - \\
KLOE16 & $-1.104(3)(2)$ & $0.142(3)\left({ }_{-4}^{+5}\right)$ & $0.073(3)\left(_{-3}^{+4}\right)$ & $0.154(6)\left({ }_{-5}^{+4}\right)$ & - \\
KLOE16 & $-1.095(3)\left({ }_{-2}^{+3}\right)$ & $0.145(3)(5)$ & $0.081(3)\left({ }_{-5}^{+6}\right)$ & $0.141(7)\left({ }_{-8}^{+7}\right)$ & $-0.044(9)\left({ }_{-13}^{+12}\right)$ \\
\hline
\end{tabular}

The smearing matrix of the $\eta \rightarrow \pi^{+} \pi^{-} \pi^{0}$ Dalitz plot is very close to diagonal. For this reason, acceptance corrected data have been used to directly fit Eq. (3). The extracted parameters are in agreement with the values obtained using the whole smearing matrix. Dalitz plot acceptance corrected data is provided as supplementary material in [15].

The unbinned integrated left-right $\left(A_{L R}\right)$, quadrant $\left(A_{Q}\right)$ and sextant $\left(A_{S}\right)$ charge asymmetries provide a more sensitive test of $C$ parity conservation with respect to the fit to the Dalitz plot. The values extracted from the analysis of the new KLOE data set are consistent with zero at $10^{-4}$ level, thus improving existent evaluations [18-20]. Systematic uncertainties are of the same size of the statistical ones except for $A_{L R}$, where the error is dominated by the description of the Bhabha background. Experimental results are reported in Tab. 2. 
Table 2. Measurements of the charge asymmetries for $\eta \rightarrow \pi^{+} \pi^{-} \pi^{0}$.

\begin{tabular}{lccc}
\hline Experiment & $A_{L R}\left(10^{-2}\right)$ & $A_{Q}\left(10^{-2}\right)$ & $A_{S}\left(10^{-2}\right)$ \\
\hline Gormley68 & $1.5 \pm 0.5$ & - & $0.5 \pm 0.5$ \\
Layter72 & $-0.05 \pm 0.22$ & $-0.07 \pm 0.22$ & $0.10 \pm 0.22$ \\
Jane74 & $0.28 \pm 0.26$ & $-0.30 \pm 0.25$ & $0.20 \pm 0.25$ \\
KLOE08 & $0.09 \pm 0.10_{-0.14}^{+0.09}$ & $-0.05 \pm 0.10_{-0.05}^{+0.03}$ & $0.08 \pm 0.10_{-0.13}^{+0.08}$ \\
KLOE16 & $-0.050 \pm 0.045_{-0.11}^{+0.05}$ & $0.020 \pm 0.045_{-0.023}^{+0.048}$ & $0.004 \pm 0.045_{-0.035}^{+0.033}$ \\
\hline
\end{tabular}

\section{Dalitz decays of the $\phi$ meson}

Vector Meson Dominance (VMD) model fails to describe the vector to pseudoscalar transition form factor (TFF) for the process $\omega \rightarrow \pi^{0} \mu^{+} \mu^{-}$, as measured by the NA60 collaboration [21]. The only other existing experimental result for $V P \gamma^{*}$ TFF comes from the SND experiment, which has measured the $\mathrm{M}_{e e}$ invariant mass distribution of the $\phi \rightarrow \eta e^{+} e^{-}$decay on the basis of 213 events [22], providing too large statistical error to confirm the NA60 evidence. New measurements of $V P \gamma^{*}$ transitions are therefore needed. A detailed study of the $\phi \rightarrow \eta e^{+} e^{-}$and $\phi \rightarrow \pi^{0} e^{+} e^{-}$decays has been performed with $1.7 \mathrm{fb}^{-1}$ of KLOE data [23, 24].

\subsection{The $\phi \rightarrow \eta e^{+} e^{-}$decay}

The $\phi \rightarrow \eta e^{+} e^{-}$decay has been studied using the $\eta \rightarrow \pi^{0} \pi^{0} \pi^{0}$ final state. Preselection cuts require: (i) two tracks of opposite sign originated from the interaction point (IP) plus six prompt photon candidates; (ii) a loose cut on the six photon invariant mass: $400<M_{6 \gamma}<700 \mathrm{MeV}$; (iii) a $3 \sigma$ cut on the recoil mass against the $e^{+} e^{-}$pair, $M_{\text {recoil }}(e e)$. A residual background contamination, due to $\phi \rightarrow \eta \gamma$ events with photon conversion on beam pipe (BP) or drift chamber walls (DCW), is rejected by extrapolating back to $\mathrm{BP} / \mathrm{DCW}$ surfaces the tracks of the $e^{+}, e^{-}$candidates and then reconstructing their invariant mass and distance. Both quantities are small for the events coming from photon conversion. $\phi \rightarrow K \bar{K}$ and $\phi \rightarrow \pi^{+} \pi^{-} \pi^{0}$ events surviving analysis cuts have more than two pions in the final state. They are rejected using time-of-flight to the calorimeter. When an EMC cluster is connected to a track, the arrival time to the calorimeter is evaluated both with calorimeter $\left(T_{\text {cluster }}\right)$ and drift chamber $\left(T_{\text {track }}\right)$ information. Events with an $e^{+}, e^{-}$candidate outside a $3 \sigma$ 's window on

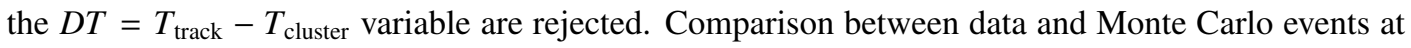
different steps of the analysis is reported in Fig. 3.

At the end of the analysis chain, 30,577 events are selected, with a residual background contamination of $\sim 3 \%$. After background subraction, the measured branching fraction for the $\phi \rightarrow \eta e^{+} e^{-}$ process is:

$$
B R\left(\phi \rightarrow \eta e^{+} e^{-}\right)=(1.075 \pm 0.007 \pm 0.038) \times 10^{-4},
$$

much more precise compared with the present PDG average of $(1.15 \pm 0.10) \times 10^{-4}$.

The slope of the transition form factor, $b_{\phi \eta}$, has been obtained from a fit to the di-lepton invariant mass using the differential cross section from Ref. [25] and the transition form factor in one-pole parametrization:

$$
F\left(q^{2}\right)=\frac{1}{1-q^{2} / \Lambda^{2}},
$$

where the slope parameter is defined as $b=\left.\left[d F\left(q^{2}\right) / d q^{2}\right]\right|_{q^{2}=0}=\Lambda^{-2}$. This results in:

$$
b_{\phi \eta}=\left(1.17 \pm 0.10_{-0.11}^{+0.07}\right) \mathrm{GeV}^{-2}
$$



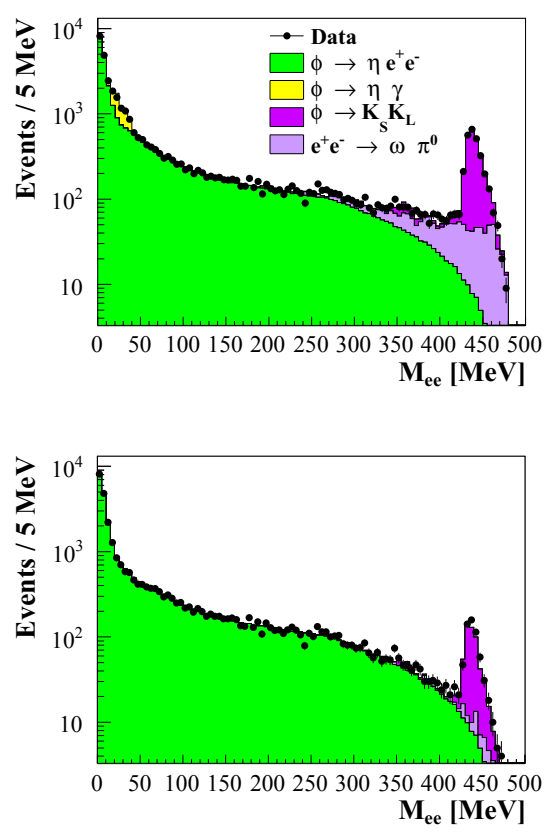
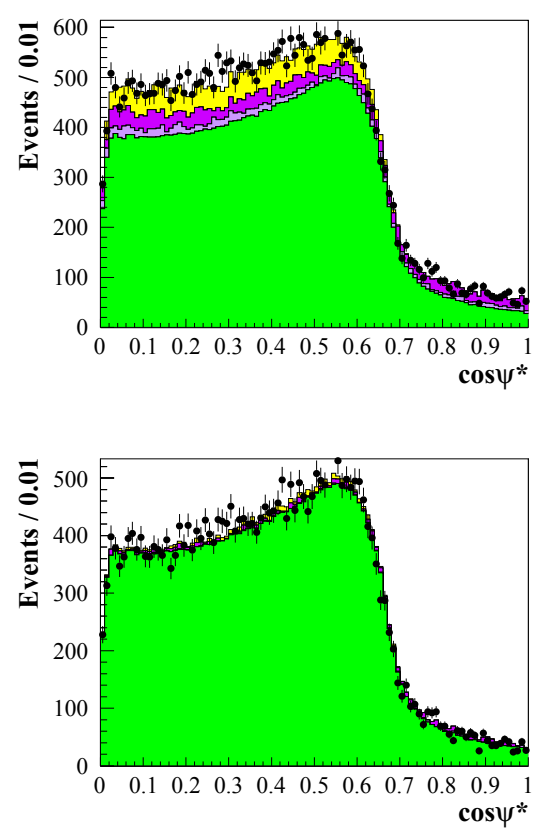

Figure 3. Analysis of the $\phi \rightarrow \eta e^{+} e^{-}$decay channel: data-MC comparison for the di-lepton invariant mass (left) and the $\cos \left(\psi^{*}\right)$ variable (right), after the cut on $M_{\text {recoil }}(e e)$ (top) and at the end of the analysis chain (bottom).

in agreement with VMD predictions $\left(b_{\phi \eta}=1 \mathrm{GeV}^{-2}\right)$. Fit results are reported in Fig. 4 left.

The squared modulus of the transition form factor, $\left|F_{\phi \eta}\left(q^{2}\right)\right|^{2}$, as a function of the $e^{+} e^{-}$invariant mass (Fig. 4 right) has been obtained by dividing the $M_{e e}$ spectrum bin by bin with the corresponding distribution obtained for MC events generated with a constant transition form factor. The value of $b_{\phi \eta}$ extracted from the fit is in agreement with Eq. (6).

\subsection{The $\phi \rightarrow \pi^{0} e^{+} e^{-}$decay}

No data are available on the transition form factor of the $\phi \rightarrow \pi^{0} e^{+} e^{-}$decay. Differently from the $\phi \rightarrow \eta e^{+} e^{-}$channel, a large background contamination is still present after preselection cuts, requiring two tracks and two photon candidates in the final state. Dedicated analysis cuts strongly reduce the main background component of Bhabha scattering events to $\sim 20 \%$, which dominates for $M_{e e}>300$ $\mathrm{MeV}$ (Fig. 5 left). The other residual relevant background contribution is from $\phi$ radiative decays. At the end of the analysis, about 14,500 events are selected, with a total background contamination of $\sim 30 \%$. Data-MC comparison is shown in Fig. 5 for different kinematical variables. The background contribution is removed bin-by-bin by subtracting the fits to each single background component from data points in the $M_{\text {recoil }}(e e)$ distribution (Fig. 6 left).

The branching ratio of the available $q^{2}$ range has been obtained from the background subtracted $e^{+} e^{-}$mass spectrum by applying an efficiency correction evaluated bin by bin:

$$
B R\left(\phi \rightarrow \pi^{0} e^{+} e^{-} ; \sqrt{q^{2}}<700 \mathrm{MeV}\right)=\left(1.19 \pm 0.05_{-0.10}^{+0.05}\right) \times 10^{-5}
$$



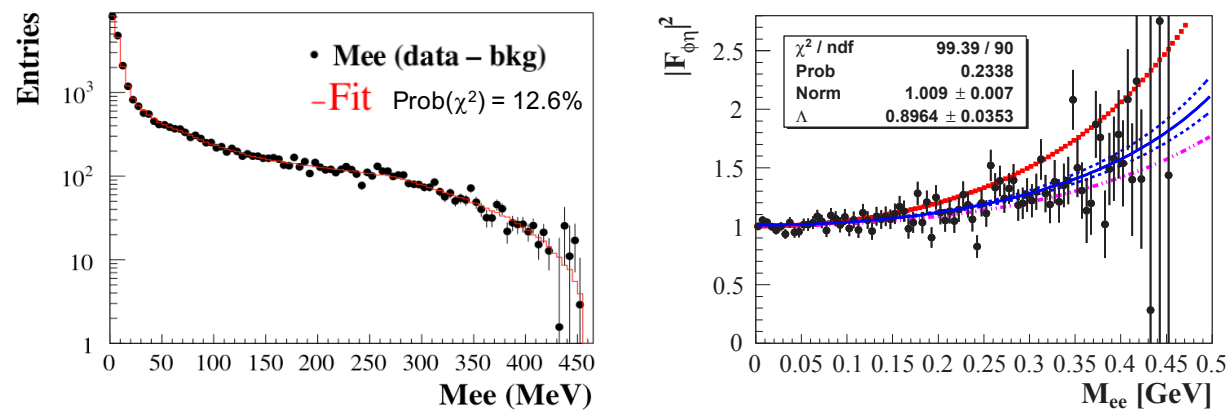

Figure 4. Left: fit to the di-lepton invariant mass for the $\phi \rightarrow \eta e^{+} e^{-}$decay channel. Right: $\phi \eta$ form factor as a function of the di-lepton invariant mass. The blue curve is the fit result, with its uncertainty; expectations from VMD and Ref. [26] are reported in red and pink, respectively.
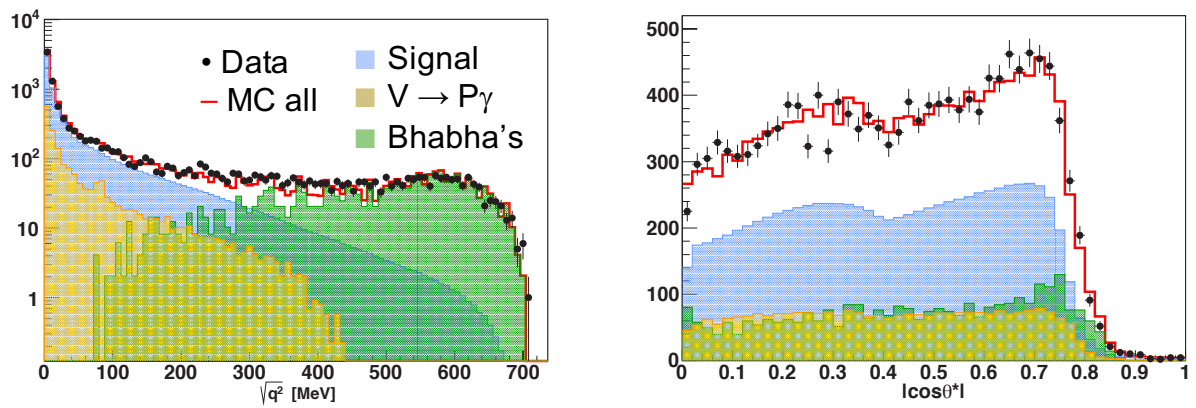

Figure 5. Analysis of the $\phi \rightarrow \pi^{0} e^{+} e^{-}$decay channel: data-MC comparison for the di-lepton invariant mass (left) and the $\cos \left(\psi^{*}\right)$ variable (right) at the end of the analysis chain.

The first error includes the statistical and normalization errors, while the second one is due to systematics on analysis cuts and background subtraction. This result can be extrapolated to the full $\sqrt{q^{2}}$ range by using the teoretical description of the decay that best fit our transition form factor data [27]:

$$
B R\left(\phi \rightarrow \pi^{0} e^{+} e^{-}\right)=\left(1.35 \pm 0.05_{-0.10}^{+0.05}\right) \times 10^{-5} .
$$

The di-lepton invariant mass after background subtraction and efficiency correction is reported in Fig. 6 right and compared with theoretical predictions [27-29]. The slope of the transition form factor has been extracted by fitting this curve in the one-pole appoximation: $b_{\phi \pi^{0}}=(2.02 \pm 0.11) \mathrm{GeV}^{-2}$.

\section{Perspectives from $e^{+} e^{-}$interactions with KLOE-2 data}

The KLOE-2 detector is taking data at the update DAФNE $e^{+} e^{-}$collider, aiming to collect $5 \mathrm{fb}^{-1}$ at the $\phi$ peak. This larger data sample will allow a deeper investigation of ligth meson properties, decay dynamics and transition form factors. About $1.5 \times 10^{10} \phi$ mesons will be produced, which will be used to complete and extend the study of the transition form factors from the Dalitz decays of vector 

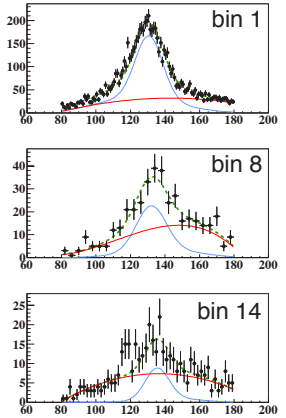
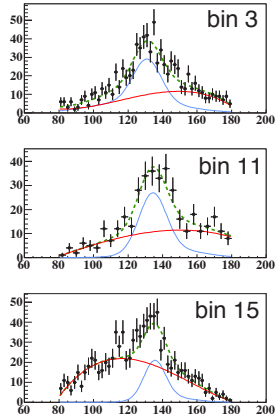

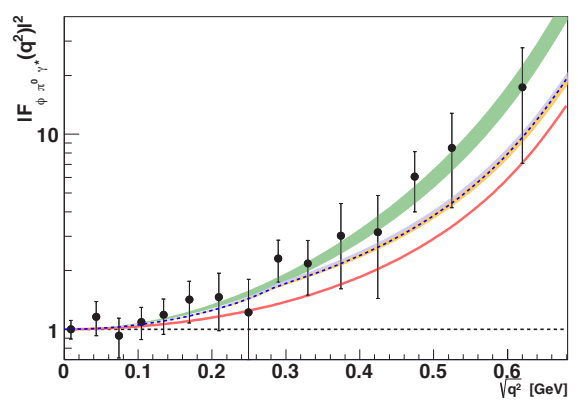

Figure 6. Analysis of the $\phi \rightarrow \pi^{0} e^{+} e^{-}$decay channel. Left: fit to the $\mathrm{M}_{\text {recoil }}(e e)$ distributions for different $\mathrm{M}_{e e}$ values, used to extract signal (blue) and background (red) components. The green lines represent the sum of the two. Right: $\phi \pi^{0}$ form factor as a function of the di-lepton invariant mass. Predictions from dispersive analyses $[28,29]$ (orange, light blue, blue dashed), chiral theory approach [27] (green) and the one-pole VMD model (red) are also reported.

mesons. As an example, the first evidence of the $\phi \rightarrow \eta \pi^{+} \pi^{-}$and $\phi \rightarrow \eta \mu^{+} \mu^{-}$decays are expected at KLOE-2. Currently, both decays have been searched by the CMD-2 experiments, that set an upper limit of [30, 31]: $B R\left(\phi \rightarrow \eta \pi^{+} \pi^{-}\right)<1.8 \times 10^{-5} @ 90 \%$ C.L., $B R\left(\phi \rightarrow \eta \mu^{+} \mu^{-}\right)<9.4 \times 10^{-6} @ 90 \%$ C.L.

A sample of $\sim 2.5 \times 10^{6} \omega$ mesons will be also produced through the Initial State Radiation

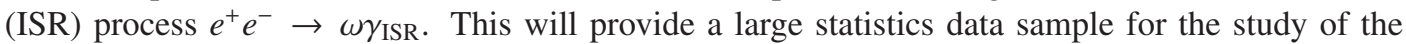
$\omega \rightarrow \pi^{+} \pi^{-} \pi^{0}$ decay dynamics. The peak obtained with $300 \mathrm{pb}^{-1}$ of KLOE data is visible in Fig. 7 left. It has been selected by requiring two track and three photons in the final state and applying a kinematic fit. No specific background rejection has been performed.

Radiative decays of the $\phi$ mesons will provide the largest data set of $\eta$ meson: additional $2.5 \times 10^{8}$ will be produced in $5 \mathrm{fb}^{-1}$. A very clean $\eta$ sample is tagged by means of the monocromatic recoil photon, $363 \mathrm{MeV}$, which is clearly identified as the most energetic neutral cluster in case of three or more particles in the final state (see Fig. 7 right). This data will be used to improve existing limits on $\eta$ violating modes, listed in Tab. 3. Existing limits and expectations combining KLOE and KLOE-2 data are also reported.

Table 3. Current upper limit for $\eta$ violating modes and extrapolations for KLOE plus KLOE-2 data set.

\begin{tabular}{lccccc}
\hline Decay & Test & UL [32] & UL (KLOE) & KLOE statistics & Scaled @ 5+2 $\mathrm{fb}^{-1}$ \\
\hline$\eta \rightarrow \gamma \gamma \gamma$ & $C$ violation & $1.6 \times 10^{-5}$ & $1.6 \times 10^{-5}$ & $410 \mathrm{pb}^{-1}$ & $3.9 \times 10^{-6}$ \\
$\eta \rightarrow \pi^{+} \pi^{-}$ & $P, C P$ violation & $1.3 \times 10^{-5}$ & $1.3 \times 10^{-5}$ & $350 \mathrm{pb}^{-1}$ & $2.9 \times 10^{-6}$ \\
$\eta \rightarrow \pi^{0} \pi^{0}$ & $P, C P$ violation & $3.5 \times 10^{-4}$ & - & & \\
$\eta \rightarrow \pi^{0} \pi^{0} \gamma$ & $C$ violation & $5.0 \times 10^{-4}$ & - & & \\
\hline
\end{tabular}

The $\eta \rightarrow \pi^{0} \gamma \gamma$ decay is an important test of $\chi_{P T}$ because of its sensitivity to $p^{6}$ on both the branching ratio (BR) and the $M_{\gamma \gamma}$ spectrum. [33, 34]. The most accurate determination of the branching fraction has been obtained by the Crystal Ball experiment at MAMI [35], which measured $B R\left(\eta \rightarrow \pi^{0} \gamma \gamma\right)=(25.2 \pm 2.5) \times 10^{-5}$. A preliminary KLOE measurement [36], based on 70 signal events, $3 \sigma$ signal evidence, provided a $4 \sigma$ lower value: $B R\left(\eta \rightarrow \pi^{0} \gamma \gamma\right)=\left(8.4 \pm 2.7_{\text {stat }} \pm 1.4_{\text {syst }}\right) \times 10^{-5}$. The completely different environment of KLOE/KLOE-2 with respect to all other $\eta \rightarrow \pi^{0} \gamma \gamma$ measure- 

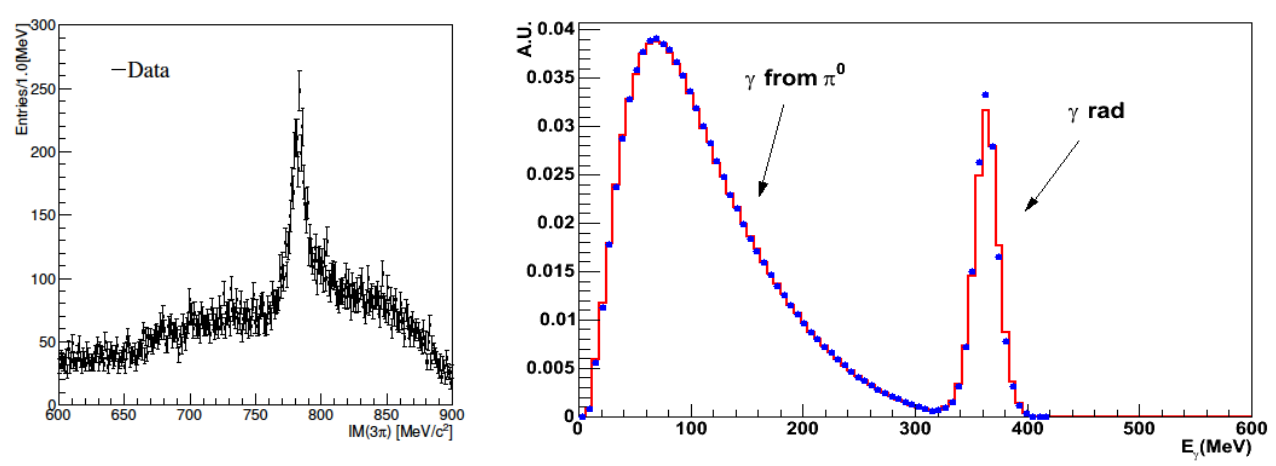

Figure 7. Left: peak of the $\omega$ meson produced through $e^{+} e^{-} \rightarrow \omega \gamma_{\text {ISR }}$ interactions. Right: photon energy for the $\phi \eta \gamma, \eta \rightarrow \pi^{0} \pi^{0} \pi^{0}$ decay. Blue dots are data while the red solid line represent MC expectations. Photons from $\pi^{0}$ 's are clearly separated from the primary photon.

ments, performed at hadron machines, asks for an upated measurement with larger statistics. using KLOE BR, a signal events comparable with Crystal Ball ( 1200 signal events) is expected merging KLOE and KLOE-2 statistics. This measurement will benefit of the new CCALT calorimeter, which is design to detect photons from the iteraction region produced at low polar angle. It will substantially improve the rejection of the most relevant background, $\eta \rightarrow \pi^{0} \pi^{0} \pi^{0}$ with merged/lost photons. MC simulation shows that $83 \%$ of the residual $\eta \rightarrow 3 \pi^{0}$ events has at least one photon lost. Half of them has photons in CCALT acceptance. KLOE/KLOE-2 statistics can provide the di-photon invariant mass to test theoretical models. As an example, a $3 \sigma$ separation is expected in the VMD $+a_{0}$ model in case of destructive and constructive interference.

KLOE-2 can improve the knowledge of $\eta$ decays in four charged particles, listed in Tab. 4. These decays probe the structure of the $\eta$ meson [25] and test the theoretical predictions of the branching ratio [39-42]. Moreover, the $\pi^{+} \pi^{-} e^{+} e^{-}$final state could reveal CP violation beyond the prediction of the Standard Model by measuring the angular asymmetry $A_{\phi}$ between pions and electrons decay planes [43]. KLOE has already measured the first two processes of Tab. 4, obtaining the most precise measurement of the BRs and the first measurement of $A_{\phi}$ with a statistical sensitivity of $2.5 \times 10^{2}$ $[37,38]$. The larger KLOE-2 data set, together with the increased track acceptance of the Inner Tracker, will improve all these measurements, allowing also to get the first evidence for the decay $\eta \rightarrow \mu^{+} \mu^{-} e^{+} e^{-}$.

Table 4. Measured and theoretical branching fractions for eta decays in four charged particles.

\begin{tabular}{lcc}
\hline Decay & $B R_{\exp }$ & $B R_{\text {theory }}$ \\
\hline$\eta \rightarrow \pi^{+} \pi^{-} e^{+} e^{-}$ & $(26.8 \pm 1.1) \times 10^{-5}$ & $(25.7 \div 30) \times 10^{-5}$ \\
$\eta \rightarrow e^{+} e^{-} e^{+} e^{-}$ & $(2.4 \pm 0.2) \times 10^{-5}$ & $(2.5 \div 2.6) \times 10^{-5}$ \\
$\eta \rightarrow \mu^{+} \mu^{-} e^{+} e^{-}$ & $B R<1.6 \times 10^{-4} @ 90 \%$ C.L. & $(1.6 \div 2.2) \times 10^{-5}$ \\
$\eta \rightarrow \pi^{+} \pi^{-} \mu^{+} \mu^{-}$ & $B R<3.6 \times 10^{-4} @ 90 \%$ C.L. & $8 \times 10^{-7}$ \\
\hline
\end{tabular}

In the $\phi \rightarrow K \bar{K} \gamma$ process, never been observed, the $K \bar{K}$ system has scalar quantum numbers, so that it is expected to proceeds through the $\phi \rightarrow\left[a_{0}(980)+f_{0}(980)\right] \gamma \rightarrow K \bar{K} \gamma$ decay. KLOE searched for this channel using the $\phi \rightarrow K_{S} K_{S} \gamma \rightarrow \pi^{+} \pi^{-} \pi^{+} \pi^{-} \gamma$ decay chain. The main backgrounds are the 


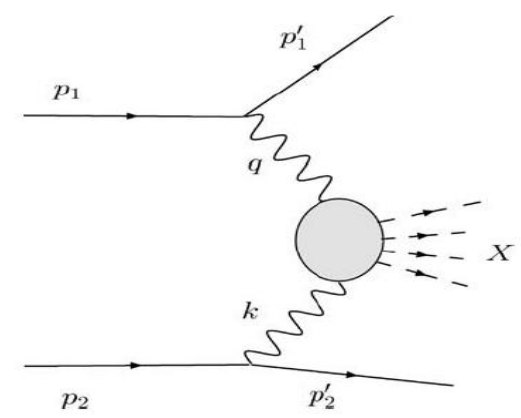

Figure 8. Diagram to produce $X$ final state through $\gamma \gamma$ interaction.

resonant $e^{+} e^{-} \rightarrow \phi \gamma \rightarrow K_{S} K_{L} \gamma$ with an ISR photon and the continuum $e^{+} e^{-} \rightarrow \pi^{+} \pi^{-} \pi^{+} \pi^{-} \gamma$ processes. MC signal has been simulated according to the phase space and the radiative decay dynamics. Selection cuts have been optimized by MC. From an integrated luminosity of $2.2 \mathrm{fb}^{-1}, 5$ candidate events are found in data, while $3.2 \pm 0.7$ background events are expected from MC. This leads to $\operatorname{BR}(\phi \rightarrow K \bar{K} \gamma)<1.9 \times 10^{-8}$ at the $90 \%$ CL [44]. This upper limit is consistent with the $\mathrm{BR}(\phi \rightarrow K \bar{K} \gamma)$ prediction computed with the $f_{0}(980)$ and $a_{0}(980)$ couplings measured by KLOE [45-47]. Scaling these numbers to the KLOE+KLOE-2 statistics a sensitivity of $B R\left(\phi \rightarrow K^{0} \bar{K}^{0} \gamma\right)<1.1 \times 10^{-8}$ is expected. The inner tracker will provide better vertex resolution, which is beneficial for the rejection capability of the background. This could lead to the first observation of such process.

\section{$5 \gamma \gamma$ interactions at KLOE and KLOE-2}

The $\gamma \gamma$ couplings and partial widths of mesons provide information about their structure and can be measured in the $e^{+} e^{-} \rightarrow e^{+} e^{-} \gamma^{*} \gamma^{*} \rightarrow e^{+} e^{-} X$ processes, Fig. 8, where $X$ is a generic $J^{P C}=0^{ \pm+}, 2^{ \pm+}$ final state. In case of a single particle $R$ produced in the final state, the cross section of the decays is proportional to the transition form factor:

$$
\sigma_{\gamma \gamma \rightarrow R}\left(q_{1}, q_{2}\right) \propto \Gamma_{R \rightarrow \gamma \gamma} \frac{8 \pi^{2}}{M_{R}} \delta\left(\left(q_{1}+q_{2}\right)^{2}-M_{R}^{2}\right)\left|F\left(q_{1}^{2}, q_{2}^{2}\right)\right|^{2} .
$$

A precise knowledge of the $\gamma \gamma$ decay width provides a measurement of the transition form factor, which is very important for the determination of the hadronic light-by-light contribution to the anomalous magnetic moment of the muon.

\subsection{Measurement of $\Gamma(\eta \rightarrow \gamma \gamma)$ at KLOE}

At KLOE, where there is no tagging of the outgoing $e^{+} e^{-}, \gamma \gamma$ interactions have been studied using off-peak data $\left(240 \mathrm{pb}^{-1}\right.$ collected at $\left.\sqrt{s}=1 \mathrm{GeV}\right)$, to avoid backgrounds from $\phi$ decays. The $\eta$ partial width, $\Gamma(\eta \rightarrow \gamma \gamma)$, is extracted from the measurement of the $e^{+} e^{-} \rightarrow e^{+} e^{-} \eta$ cross section, using both neutral and charged $\eta \rightarrow \pi \pi \pi$ decay channels [48]. The main background is due to the $e^{+} e^{-} \rightarrow \eta \gamma$ reaction, with an undetected recoil photon. After reducing the background components with specific kinematical cuts, signal events are extracted by fitting with the expected Monte Carlo components the two-dimentional plot $M_{\text {miss }}^{2}-p_{L / T}$ (Figs. 9), where $M_{\text {miss }}^{2}$ is the squared missing mass and $p_{L / T}$ is the $\eta$ 

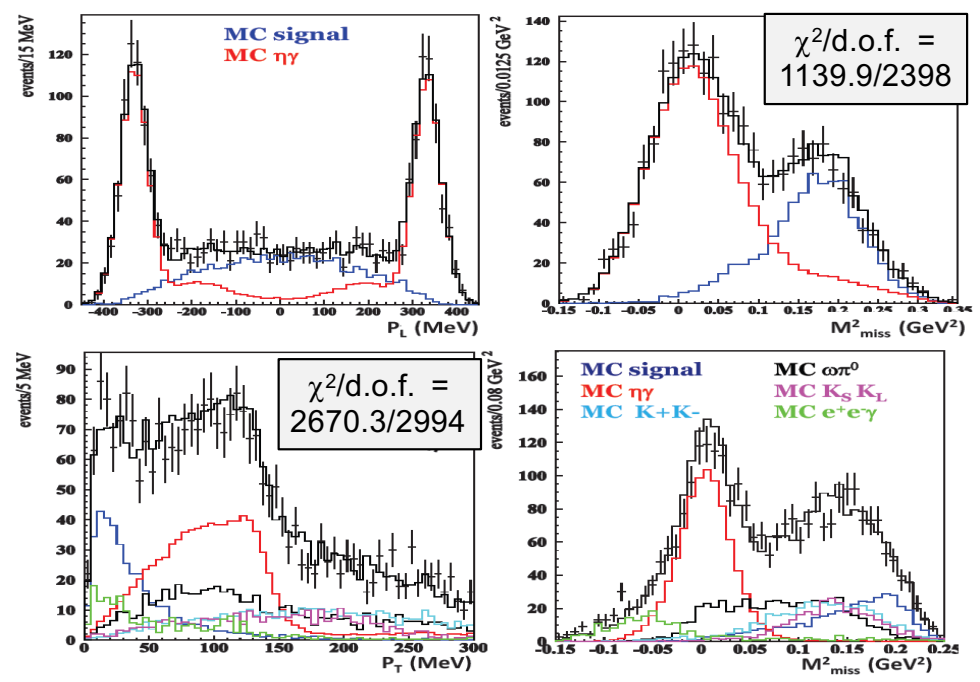

Figure 9. Top: $\eta$ longitudinal momentum (left) and $M_{\text {miss }}^{2}$ distribution (right) for $\gamma \gamma \rightarrow \eta \rightarrow \pi^{0} \pi^{0} \pi^{0}$ events. Bottom: $\eta$ tranverse momentum (left) and $M_{\text {miss }}^{2}$ distribution (right) for $\gamma \gamma \rightarrow \eta \rightarrow \pi^{+} \pi^{-} \pi^{0}$ events. Points with error bars are data, black solid histograms are fit result. Different fit components are reported in colors.

longitudinal/transverse momentum in the $\pi^{0} \pi^{0} \pi^{0} / \pi^{+} \pi^{-} \pi^{0}$ decay. Combining the two measurements, the extracted value for the production cross section results to be:

$$
\sigma\left(e^{+} e^{-} \rightarrow e^{+} e^{-} \eta\right)=\left(32.7 \pm 1.3_{\text {stat }} \pm 0.7_{\text {syst }}\right) \mathrm{pb}
$$

This value is used to extract the most precise measurement to date of the $\eta \rightarrow \gamma \gamma$ partial width:

$$
\Gamma(\eta \rightarrow \gamma \gamma)=\left(520 \pm 20_{\text {stat }} \pm 13_{\text {syst }}\right) \mathrm{eV} .
$$

\subsection{The $\pi^{0}$ Transition Form Factor at KLOE-2}

The upgrade of the KLOE-2 detector, with four detectors installed to tag electrons and positrons from the reaction $e^{+} e^{-} \rightarrow e^{+} e^{-} \gamma^{*} \gamma^{*} \rightarrow e^{+} e^{-} X$, will give the opportunity to investigate $\gamma \gamma$ physics also at the $\phi$ resonance for the reactions $\gamma \gamma \rightarrow \pi^{0} / \pi \pi / \eta / \eta \pi$ [12]. Single pseudoscalar production will improve the determination of the two-photon decay widths of these mesons, $\Gamma_{\gamma \gamma}$. For the $\pi^{0}$, the most precise measurement is obtained exploiting the Primakoff effect, reaching an accuracy of $2.8 \%$ [49]. At KLOE-2, the coincidence between the KLOE central detector and the HET taggers will provide a very clean sample of $\sim 1900 \gamma \gamma \rightarrow \pi^{0}$ events per $\mathrm{fb}^{-1}$, with background from radiative Bhabha scattering events being rejected by using the coincidence between the central detector and the HET stations. An accuracy of $1 \%$ on $\Gamma_{\gamma \gamma}\left(\pi^{0}\right)$ is reachable with 5-6 $\mathrm{fb}^{-1}$, matching the current theory precision (Fig. 10 left). With the same amount of data, the measurement of the $\pi^{0} \rightarrow \gamma \gamma^{*}$ transition form factor in the space-like region at low momentum transfer for the virtual photon will be possible with 5-6\% accuracy. The KLOE-2 measurement will cover an unexploited region of the momentum transfer, as shown in Fig. 10 right. For the form factor measurement, the coincidence between the central detector and one of the HET stations will be used. These two proposed measurements are relevant for the theoretical evaluation of the hadronic light-by-light contribution to the muon magnetic anomaly, that is limited by the knowledge of the pseudoscalar transition form factor [50]. 

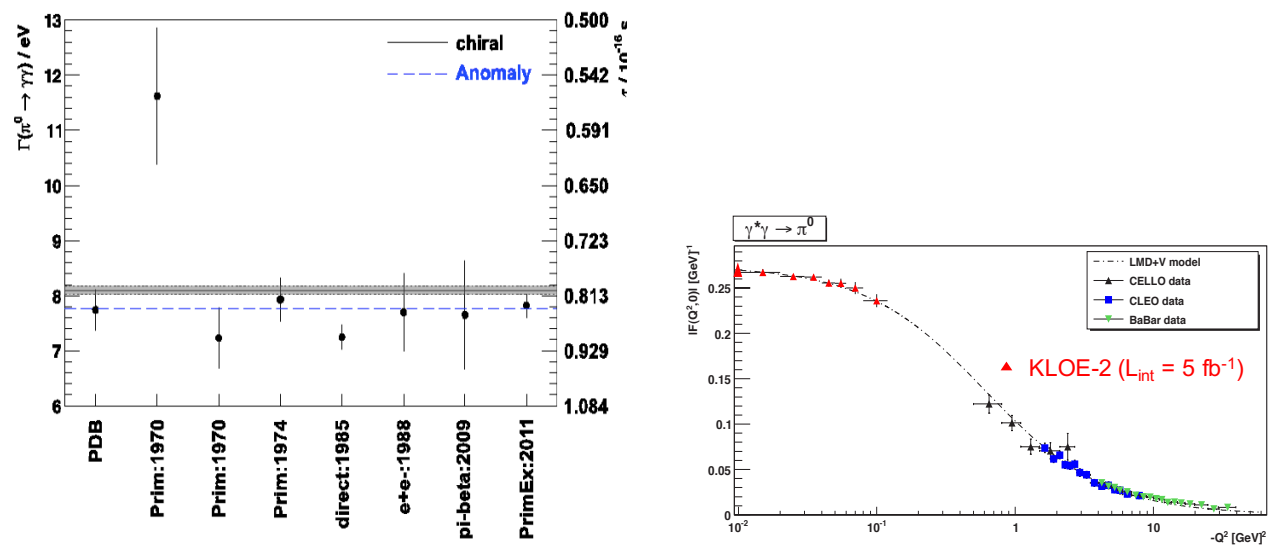

Figure 10. Left: experimental measurements of $\Gamma\left(\pi^{0} \rightarrow \gamma \gamma\right)$ (black dots) and predictions from $\chi \mathrm{PT}$ with (black line) and without (blue dotted line) chiral anomaly. The theoretical error band is displayed in grey. Right: KLOE-2 expectations for $\pi^{0} \gamma \gamma^{*}$ TFF (red triangles), compared with existing measurments from CELLO [51] (black triangles), CLEO [52] (blue squares) and Babar [53] (green triangles).

\section{Conclusions}

The large data sample of light mesons collected with the KLOE detector provided several state-ofthe-art measurements on the properties of light scalar, pseudoscalar and vector mesons. In the last year, the most precise measurements of the $\eta \rightarrow \pi^{+} \pi^{-} \pi^{0}$ decay dynamics and of the $\phi \rightarrow \eta e^{+} e^{-}$, $\phi \rightarrow \pi^{0} e^{+} e^{-}$transition form factors have been published.

Currently, the KLOE-2 run is in progress aiming to collect at least $5 \mathrm{fb}^{-1}$ within the first months of year 2018. This data sample will allow to extend the high precision investigation of light meson properties. KLOE- 2 will be an ideal tool to study in details the $\pi^{0}$ and $\eta$ transition form factor at momentum transfer below $1 \mathrm{GeV}^{2}$.

\section{References}

[1] KLOE Collaboration, LNF-92/019 (IR) (1992) and LNF-93/002 (IR) (1993)

[2] A. Gallo et al., Conf. Proc. C060626 (2006) 604, SLAC-PUB-12093

[3] M. Adinolfi et al., Nucl. Instr. Meth. A 48851 (2002)

[4] M. Adinolfi et al., Nucl. Instr. Meth. A 482364 (2002)

[5] M. Adinolfi et al., Nucl. Inst. Meth. A 492134 (2002)

[6] F. Ambrosino et al., Nucl. Inst. Meth. A 534403 (2004)

[7] C. Milardi et al., JINST 7 T03002 (2012)

[8] D. Babusci et al., Acta Phys. Polon. B 4681 (2015)

[9] A. Di Cicco et al., Acta Phys. Polon. B 4673 (2015)

[10] M. Cordelli et al., Nucl. Instr. Meth. A 718 81-82 (2013)

[11] F. Happacher et al., Acta Phys. Polon. B 4687 (2015)

[12] G. Amelino-Camelia et al., Eur. Phys. J. C 68619 (2010)

[13] H. Leutwyler, Mod. Ph. Lett. A 281360014 (2013) 1360014

[14] F. Ambrosino et al., JHEP 05006 (2008) 
[15] A. Anastasi et al., JHEP 05019 (2016)

[16] P. Adlarson et al., Phys. Rev. C 90045207 (2014)

[17] M. Ablikim et al., Phys. Rev. D 92012014 (2015)

[18] M. Gormley et al., Phys. Rev. Lett. 21402 (1968)

[19] J. G. Layter et al., Phys. Rev. Lett. 29316 (1972)

[20] M. R. Jane et al., Phys. Lett. B 48260 (1974)

[21] S. Damjanovic et al., Phys. Lett. B 677 (2009) 260.

[22] M. N. Achasov et al., Phys. Lett. B 504 (2001) 275.

[23] D. Babusci et al., Phys. Lett. B 7421 (2015)

[24] A. Anastasi et al., Phys. Lett. B 757362 (2016)

[25] L.G. Landsberg, Phys. Rep. 128301 (1985)

[26] C. Terschlusen and S. Leupold, Phys. Lett. B 691191 (2010)

[27] S. Ivashyn, Prob. Atomic. Sci. Technol. 2012N1 179 (2012)

[28] S. P. Schneider, B. Kubis, F. Niecknig, Phys. Rev. D 86054013 (2012)

[29] I. Danilkin et al., Phys. Rev. D 91094029 (2015)

[30] R. R. Akhmetshin et al., Phys. Lett. B 49181 (2000)

[31] R. R. Akhmetshin et al., Phys. Lett. B 591191 (2001)

[32] C. Patrignani et al. (Particle Data Group), Chin. Phys. C 40100001 (2016)

[33] J. Bijnens, Phys. Scripta T 9934 (2002)

[34] E. Oset, J. R. Pelaez, L. Roca, Phys. Rev. D 67073013 (2003)

[35] B. M. K. Nefkens et al., Phys. Rev. C 90025206 (2014)

[36] B. Di Micco et al., Acta Phys. Slov. 56403 (2006)

[37] F. Ambrosino et al., Phys. Lett. B 675283 (2009)

[38] F. Ambrosino et al., Phys. Lett. B 702324 (2011)

[39] C. Jarlskog and H. Pilkuhn, Nucl. Phys. B 1264 (1967)

[40] A. Faessler, C. Fuchs and M. I. Krivoruchenko, Phys. Rev. C 61035206 (2000)

[41] C. Picciotto and S. Richardson, Phys. Rev. D 483395 (1993)

[42] B. Borasoy and R. Nissler, Eur. Phys. J. A 3395 (2007)

[43] D. N. Gao, Mod. Phys. Lett. A 171583 (2002)

[44] F. Ambrosino et al., Phys. Lett. B 67910 (2009)

[45] F. Ambrosino et al., Phys. Lett. B 634148 (2006)

[46] F. Ambrosino et al., Eur. Phys. J. C 49473 (2007)

[47] F. Ambrosino et al., Phys. Lett. B 6815 (2009)

[48] D. Babusci et al., JHEP 01199 (2013)

[49] I. Larin et al., Phys. Rev. Lett. 106162303 (2011)

[50] D. Babusci et al., Eur. Phys. J. C 721917 (2012)

[51] H. J. Behrend et al., Z. Phys. C 49401 (1991)

[52] J. Gronberg et al., Phys. Rev. D 5733 (1998)

[53] (B. Aubert et al., Phys. Rev. D 80052002 (2009) 